ISSN 2073-8994

www.mdpi.com/journal/symmetry

Article

\title{
Effective Supramolecular Chirogenesis in Ethane-Bridged Bis-Porphyrinoids
}

\author{
Victor Borovkov \\ Department of Applied Chemistry, Osaka University, 2-1 Yamada-oka, Suita, Osaka 565-0871, Japan; \\ E-Mail: victrb@chem.eng.osaka-u.ac.jp
}

Received: 19 November 2009; in revised form: 26 January 2010 / Accepted: 20 February 2010 / Published: 23 February 2010

\begin{abstract}
This feature article gives a general introduction to the phenomenon of supramolecular chirogenesis using the most representative examples of different chirogenic assemblies on the basis of ethane-bridged bis-porphyrinoids. Supramolecular chirogenesis is based upon a smart combination of supramolecular chemistry and chirality sciences and deals with various aspects of asymmetry induction, transfer, amplification, and modulation. These chiral processes are governed by numerous noncovalent supramolecular forces thus allowing a judicious, mechanistic, and dynamic control by applying a variety of internal and external influencing factors. Currently, supramolecular chirogenesis is widely used in different fields of fundamental and applied branches of science and modern technology, touching on such important issues as origin of chirality on the Earth, asymmetry sensing, enantioselective catalysis, nonlinear optics, polymer and materials science, pharmacy and medicine, nanotechnology, molecular and supramolecular devices, chiral memory, absolute configuration determination, etc.
\end{abstract}

Keywords: porphyrin; chirality; chirogenesis; supramolecular chemistry; circular dichroism

\section{Introduction}

Chirality in general is an ability of some objects to exist as a pair of non-superimposable mirror images (Figure 1). In chemistry this phenomenon most often relates to molecules and (supra) molecular systems, atoms or other components that are asymmetrically arranged in three spatial dimensions around a center, axis, or plane. As the simplest example of chemical chirality, a tetrahedral $\mathrm{sp}^{3}$ carbon atom having four different substituents can be adequately referred. Natural amino acids, 
with the exception of glycine, are the most commonly occurring chemical compounds exhibiting this type of chirality [1]. Besides this classical case, there are more complicated kinds of chirality associated with the corresponding conformational changes of a particular part of a molecule or the whole molecule, or assembly of molecules under a certain chiral influence, thus resulting in the formation of an asymmetrical structure. With supramolecular interactions being responsible for such chiral transformation, all the phenomena dealing with various aspects of asymmetry induction, transfer, amplification and modulation come under the heading of supramolecular chirality or more specifically supramolecular chirogenesis [2-7]. These chirogenic effects are abundant in many natural systems such as the DNA double helix, the secondary $\alpha$-helix structure of proteins, photosynthetic apparatus, etc. [1,8-10]. Besides, there are numerous artificially made supramolecular assemblies based on various classes of chemical compounds and structures exhibiting chirogenic properties. Therefore, in order to emphasize the great diversity of supramolecular chirogenic effects studied to date, the most representative examples of different non-covalent associations and host-guest assemblies, in particular on the basis of ethane-bridged bis-porphyrinoids, will be overviewed.

Figure 1. A pair of non-superimposable mirror images.
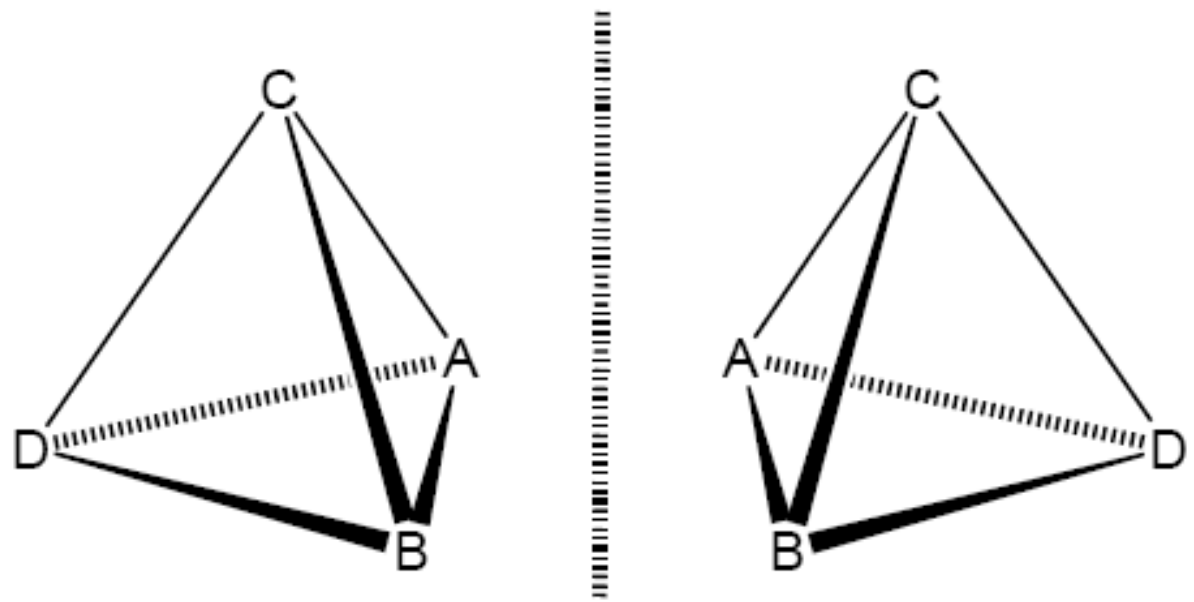

mirror plane

\section{Host-Guest Binding and Mechanism of Supramolecular Chirogenesis in Ethane-Bridged Bis-Porphyrins}

Porphyrinoids are naturally occurred tetrapyrrolic pigments, which play a decisive and vital role in many important biochemical processes, such as oxygen transport in living organisms, various enzymatic reactions, metabolic pathways, photosynthesis, etc. [1]. It has also turned out that these compounds are particularly well-suited for studying the processes of supramolecular chirogenesis, owing to their specific and highly appropriate spectral, physico-chemical and chemical properties, facile handling, and superior propensity to form various supramolecular assemblies [2-7].

One of the most comprehensive investigation of supramolecular chirogenesis in porphyrinoids to date was undertaken by our group using a series of relatively simple achiral ethane-bridged bisporphyrin hosts 1-4, as depicted in Figure 2 [2-7,11-32]. These hosts were particularly designed in order to ensure effective chirogenic properties using the most efficient structural motif for 
supramolecular chirogenesis in terms of availability, simplicity, and versatility. Essentially, the bisporphyrin systems 1-4 are able to adopt different types of asymmetric conformations and to exhibit corresponding chiroptical responses upon interaction with various classes of chiral guests, including chemical substances with different functional groups, as well as monodentate and bidentate compounds. One of the main factors affording this versatility is semi-flexibility/semi-rigidity of the ethane linkage between two porphyrin macrocycles, which is relatively short to prevent rotation of the two porphyrin subunits around the covalent bridge, but sufficiently flexible to allow the twist of two directly connected porphyrin moieties in the extended anti and tweezer conformations.

Figure 2. Schematic representation of 1-4 and corresponding conformational changes controlled by the structural type of external ligand: “face-to-face” conformation without ligand, tweezer conformation upon interaction with bidentate ligands, and linearly extended anti conformation upon interaction with monodentate ligands or excess of bidentate ligands in some cases.

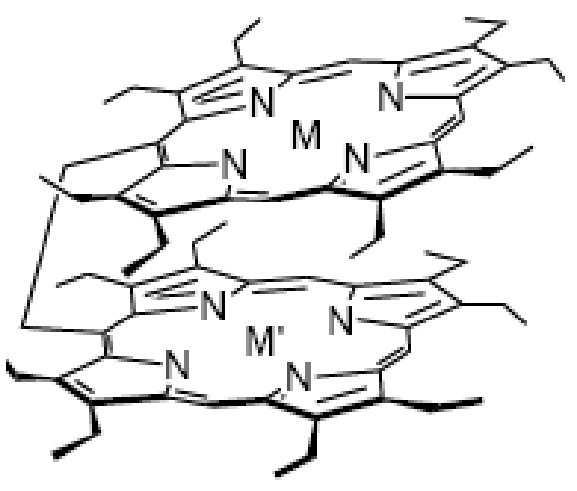

"face-to-face" conformation

l $\mathrm{M}=\mathrm{M}^{\prime}=\mathrm{Zn}$

$2 \mathrm{M}=\mathrm{M}^{\prime}=\mathrm{Mg}$

$3 \mathrm{M}=\mathrm{M}^{\prime}=2 \mathrm{H}$

$4 \mathrm{M}=2 \mathrm{H}, \mathrm{M}^{\prime}=\mathrm{Zn}$

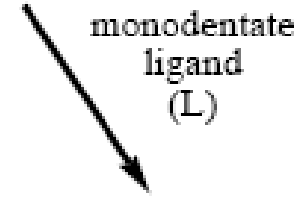

ligand

(L)

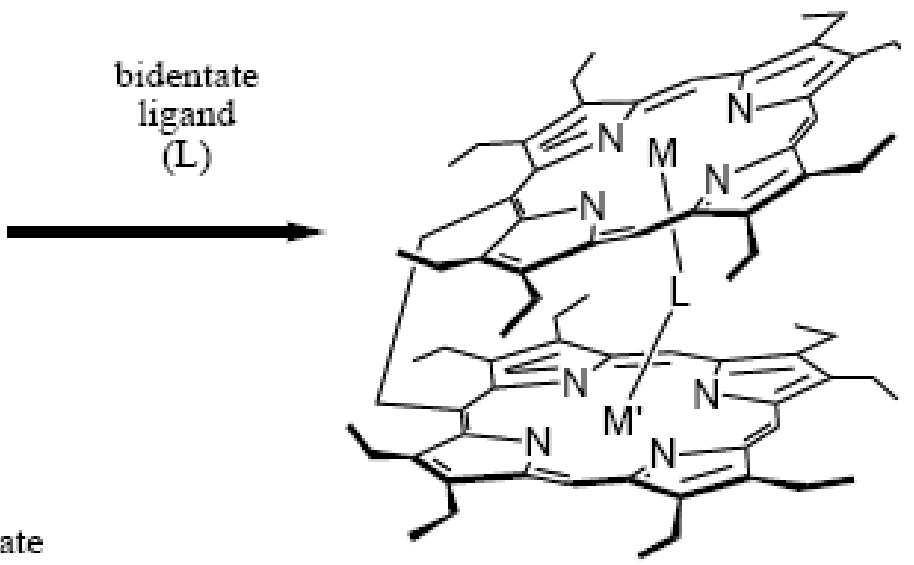

tweezer conformation (in the case of 1 )

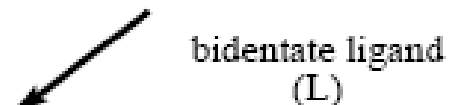

(L)

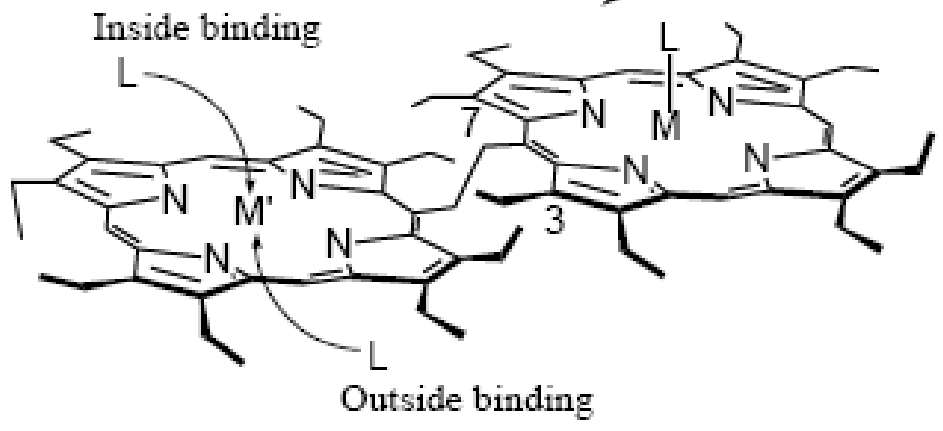

linearly extended anti conformation

Initially, the bis-porphyrins 1-4 are in a syn "face-to-face" spatial conformation in nonpolar, noncoordinating solvents owing to the strong interactions between the porphyrin subunits. Further interaction with an external guest induces a conformational rearrangement to yield the extended anti-form in the case of monodentate guests (for 1-4) or to give the tweezer structure in the case of geometrically suitable bidentate guests (for $\mathbf{1}$ ) (Figure 2). These binding events are highly cooperative 
processes resulting in stable 1:2 or 1:1 host-guest complexes, respectively, in the case of bis-porphyrin 1. As these events take place, the Gibbs free energy change ranges from -6.7 to $-8.4 \mathrm{kcal} \mathrm{mol}^{-1}$ for primary amines as monodentate guests, and being as great as $-9.5 \mathrm{kcal} \mathrm{mol}^{-1}$ for 1,2-diaminocyclohexane (DACH) as an example of a bidentate guest. The observed structural transformations (syn-to-anti and syn-to-tweezer) are a rather general feature of this supramolecular host and take place upon binding of any type of coordinating guests regardless of their chirality, as in the case of chiral and achiral ones.

However, when a chiral compound is being used as an external guest, an additional structural deformation occurs owing to effective chirality transfer from the enantiomeric guest to the achiral bisporphyrin hosts 1-4. Particularly, in the case of chiral monodentate compounds upon inside binding (approaching from the side of the ethane bridge, see the linearly extended anti conformation in Figure 2), there are competitive steric repulsive interactions between the two most bulky substituents, such as $X$ and $X^{\prime}$ (where $X>X^{\prime}$ ), at the guest's stereogenic center *C and the ethyl groups at the 3and 7-positions of the neighboring porphyrin moiety (Figure 3). The dominant steric hindrance in this system generated by interaction of the bulkiest $\mathrm{X}$ substituent and the ethyl group at the 3-position forces the neighboring porphyrin ring to move outward. This leads to formation of a unidirectional screw twist between two porphyrin moieties in 1-4. The induced screw architecture is a nonsuperimposable mirror image and thus considered to be chiral, as a result of the chiral information transfer from the chiral guest to the achiral bis-porphyrin. The generated asymmetry in the corresponding bisporphyrin's structure is retained by another type of the steric hindrances, which arise from the repulsive interaction between the 3,7-ethyl groups of the two neighboring porphyrin subunits. This interaction makes it impossible for the two porphyrin moieties to rotate around the ethane-bridge axis, thus preventing the process of racemization of the generated screw. As a result of these chirogenic events, a rather appreciable (moderate-to-strong) circular dichroism (CD) exciton couplet signal (negative-to-positive or positive-to-negative, depending on the screw orientation) is induced in the porphyrin B (Soret) absorption region (Figure 4). The chirality sign, as determined by the sign of the first Cotton effect located at the lower energy region, unequivocally correlates with the generated helicity of the bis-porphyrin structure, which is dependent upon the guest stereochemistry, hence allowing straightforward assignment of the guest's absolute configuration. Specifically, when the bulkiness order of the guest's substituents around the stereogenic center coincides with the priority order of the relevant substituents determined by the Cahn-Ingold-Prelog rule [33], the $(R)$-enantiomer yields a negative chirality that corresponds to a negative first Cotton effect and a positive second Cotton effect, while the $(S)$-enantiomer gives a CD couplet of the opposite (positive-to-negative) signs. However, in the case of chiral guest, the substituent size order of which does not correspond with the priority order according to the Cahn-Ingold-Prelog rule, the opposite chirality sign is observed for the same enantiomer, that is the $(R)$-enantiomer induces a positive chirality, while the $(S)$-enantiomer generates a negative chirality. This straightforward correlation between the induced chirality sign and the stereochemical information of interacting guests makes this sensing protocol highly suitable for determining the absolute configuration of different classes of chemical compounds. 
Figure 3. Schematic representation of the steric mechanism of chirality induction in the anti conformation of 1-4 upon interaction with monodentate guests.

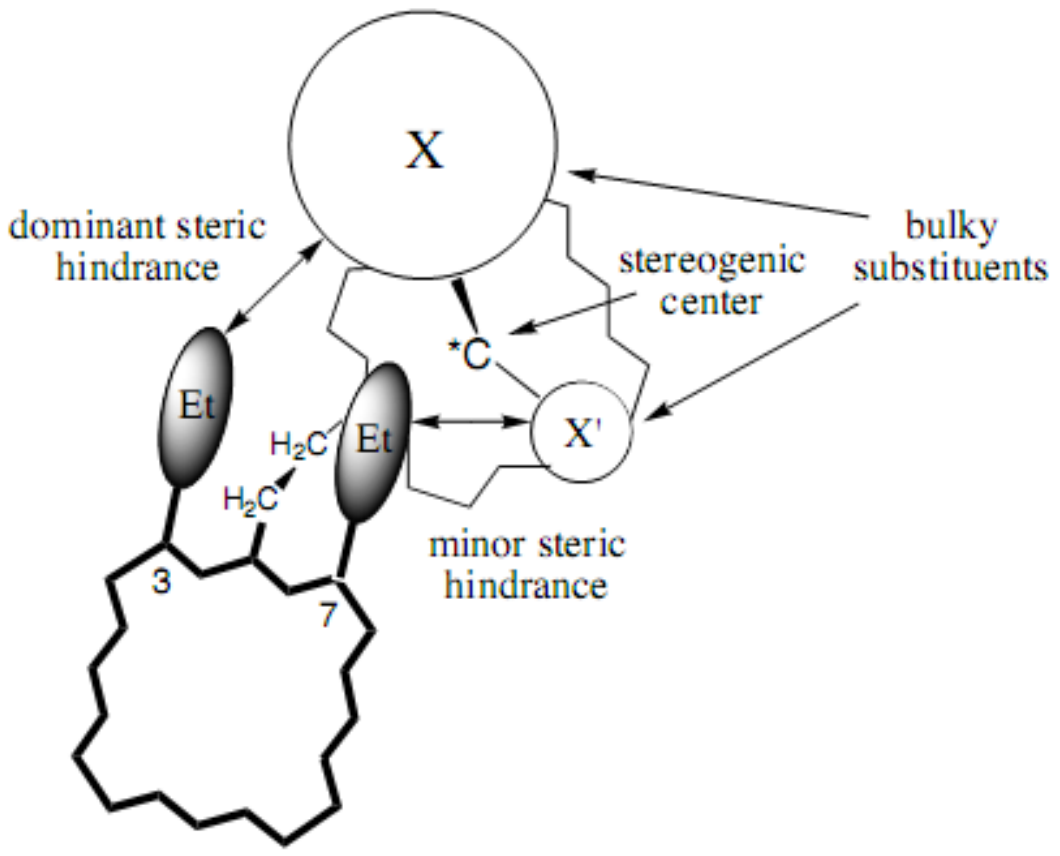

left-handed screw structure induced by $(R)$-guest

Figure 4. Schematic representation of the induced positive-to-negative and negative-topositive CD couplets.

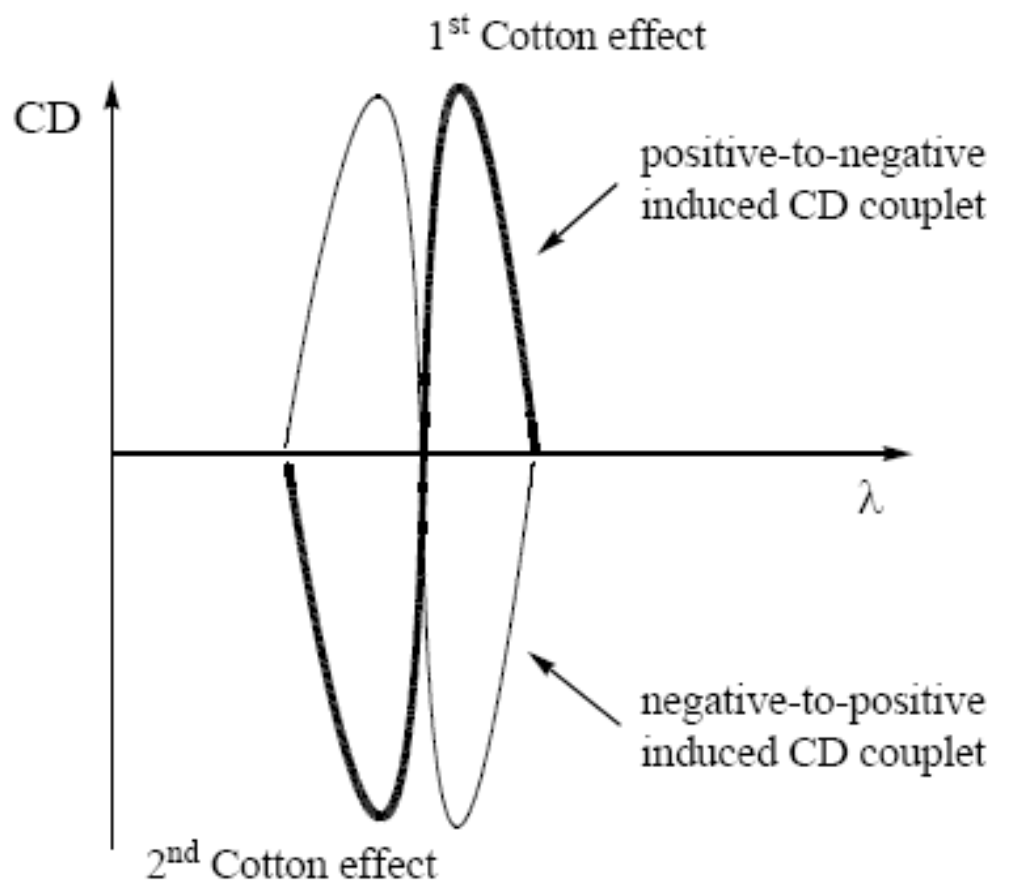

The electronic mechanism of chirality induction is based upon the spatial arrangement of the lowest energy Soret band transitions of the porphyrin moieties in the corresponding bis-porphyrin, which are orientated along to the axis connecting the 5- and 15-positions of the porphyrin rings (or respectively 
in the parallel orientation to the ethane bridge in the linearly extended anti conformation). In the case of $(R)$-enantiomers, the relative orientation between these two electronic transitions of the neighboring porphyrin moieties is anticlockwise, which according to the exciton chirality method [34] corresponds to negative chirality, whilst for (S)-enantiomers the orientation of these transitions is opposite (clockwise), thus resulting in the positive sign of the induced chirality (Figure 5). This comprehensive rationalization of the host-guest interaction and chirality induction mechanisms allowed not only the development of a smart and universal chirality sensor, which can be used for straightforward determination of the absolute configuration of various chiral guests, but also the detailed investigation of various external and internal factors controlling the chirogenic properties in these supramolecular systems.

Figure 5. Schematic representation of the electronic mechanism of chirality induction in the anti conformation of 1-4 upon interaction with monodentate guests.

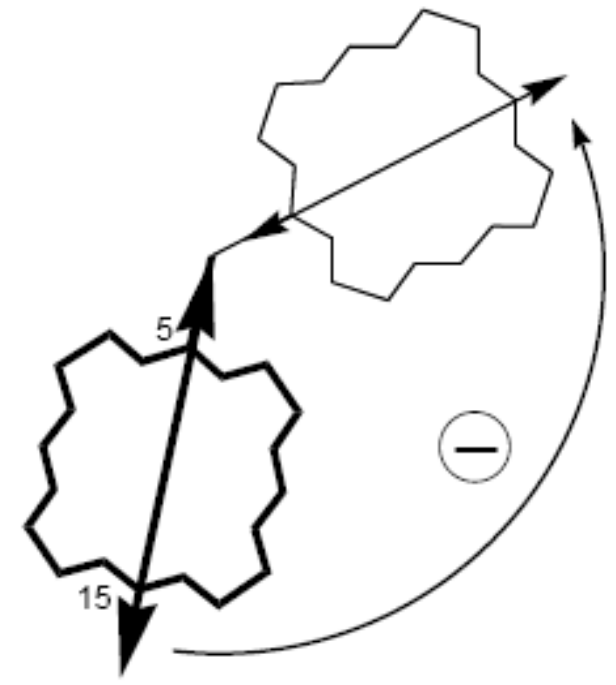

left-handed screw structure induced by $(R)$-guest

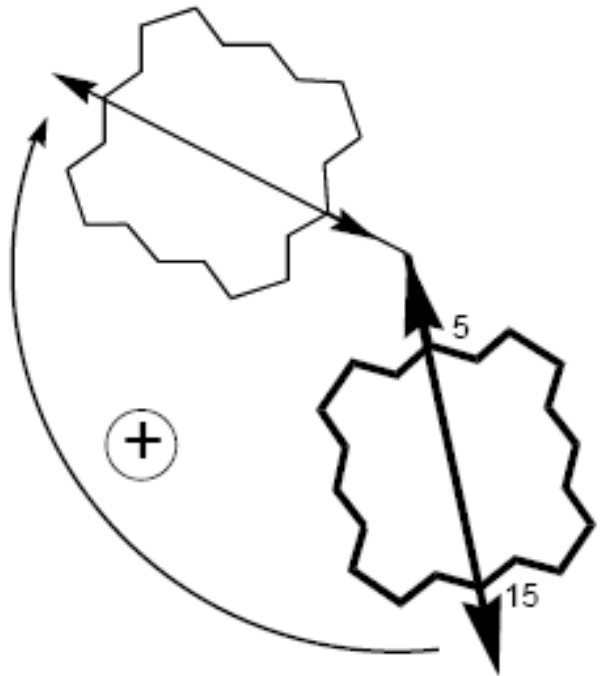

right-handed screw structure induced by $(S)$-guest

\section{External and Internal Factors Controlling Supramolecular Chirogenesis in Ethane-Bridged Bis-Porphyrins [2-7,11-32]}

One of the most important factors controlling supramolecular chirogenesis is a number of binding sites for the corresponding host-guest interactions. As stated above, due to the dynamic nature of these supramolecular systems, there are two basic modes of binding for the chiral (and for achiral as well) guests in 1-4; i.e., inside binding and outside binding (Figure 2), yielding a number of possible conformations. In the case of inside binding, the host-guest interactions occur from the side that results in a close spatial proximity of the bulky chirality-determining $X$ and $X$ ' substituents at the stereogenic center of the guest to the ethyl groups at the 3- and 7-positions of the adjacent porphyrin moiety (Figure 3), thus allowing chiral supramolecular steric interactions. In the case of outside binding, it is apparent that these chirogenic interactions cannot occur because of the distant location of the guest. The presence of these steric interactions was clearly demonstrated by comparison of the difference in structural rigidity between the fully ligated $\mathbf{1}$ and $\mathbf{4}$. This was determined by the full width at half- 
maximum (fwhm) values of the corresponding absorption spectra: the smaller the fwhm value, the more rigid the structure. Obviously, the presence of only one binding site in $\mathbf{4}$ results in the lower percentage of possible conformations exhibiting these steric chirogenic interactions in comparison to $\mathbf{1}$ possessing two binding sites. Therefore, in general the host-guest systems on the basis of $\mathbf{4}$ should be more flexible, thus producing greater the fwhm values in comparison to those of $\mathbf{1}$. In turn, this greater number of chirogenic interactions and consequently enhanced rigidity directly translates into an increase of circa $250 \%$ in supramolecular chirogenesis for the host-guest systems based on $\mathbf{1}$ over the corresponding assemblies on the basis of 4 .

Another key factor controlling supramolecular chirogenesis is the bulkiness effect. As stated above, the degree of the induced screw in 1-4 is dependent upon the difference between the magnitudes of the steric repulsions caused by the guest substituents ( $\mathrm{X}$ and $\mathrm{X}^{\prime}$ ) at the stereogenic center (Figure 3). The crucial importance of the substituent's steric effect is exemplified by the distance dependence of the chiral center to the binding site. Thus, when the chiral center of the guest is at a position $\beta$ to the binding group, the asymmetrically orientated $X$ and $X$ ' substituents are further apart from 3,7-ethyl groups. This diminishes the steric repulsion effect felt by the neighboring porphyrin moiety, hence reducing the degree of the induced screw and subsequently inducing smaller CD signals in $\mathbf{1}$. The bulkiness effect has been further investigated by varying the size of the guest. As the size of the bulkiest $\mathrm{X}$ substituent of the ligand is increased, whilst the size of the second bulky $\mathrm{X}^{\prime}$ 'substituent remains unchanged, the dominant steric hindrance between the $\mathrm{X}$ substituent and the 3-ethyl group is enhanced (Figure 6). In order to minimize this repulsive interaction, the screw angle between the two porphyrin moieties increases resulting in formation of the greater screw structure and subsequently yielding the more intense exciton couplet CD signal. Importantly, it was shown that the total CD amplitude is linearly dependent upon the size of the largest $\mathrm{X}$ substituent at the stereogenic center $\left({ }^{*} \mathrm{C}\right)$ for homologous ligands, hence making it possible to predict the magnitude of induced chirality, if the chirality is known, for example, the chirogenic effect is a direct measure of the relative bulkiness of the largest substituent.

The medium in which the chirogenic processes take place is often overlooked as a significant contributing factor. However, it has been found that chiroptical properties of supramolecular systems can be profoundly affected by the solvent used. Hence, further study of the bulkiness effect revealed a decisive role of solvent as an active part of the overall supramolecular system that considerably influences the chiroptical properties, sometimes leading even to such intriguing phenomenon as chirality inversion. In particular, it was realized through judicious analysis of the selective solutesolvent interactions especially in the borderline cases where the differences between the competitive chirogenic interactions were small and the substituent's relative size could be modulated upon formation of a specific solvation shell. For example, in the case of the guest having two substituents (X and $\left.\mathrm{X}^{\prime}\right)$ at the stereogenic center $\left({ }^{*} \mathrm{C}\right)$ of similar sizes, with the non polar $\mathrm{X}$ group of slightly larger size in comparison to the polar $\mathrm{X}^{\prime}$ group, the dominant steric hindrance between the larger $\mathrm{X}$ substituent and the 3-ethyl group induces the conventional left-handed screw structure in non polar, non interacting solvents, thus resulting in a negative exciton couplet CD signal (Figure 7). However, in polar media, the solvent molecules are able to dipole-dipole interact with the polar $\mathrm{X}^{\prime}$ group, thus forming a solvent shell and increasing the corresponding size of this group. This switches the dominant stereogenic interactions from the $\mathrm{X} / 3$-Et pair to the $\mathrm{X}^{\prime} / 7-\mathrm{Et}$ pair that results in shifting the screw 
orientation to right-handed and consequently the chirality inversion to generate a positive exciton couplet CD signal. This solvent effect was nicely exemplified by a nonlinear dependence of the induced chirality upon the solvent composition consisted of polar and non polar components, thus revealing the presence of highly selective solvent-solute interactions.

Figure 6. Schematic representation of the bulkiness effect on the magnitude of the supramolecular chirogenesis in the anti conformation of $\mathbf{1}$ upon interaction with monodentate guests.

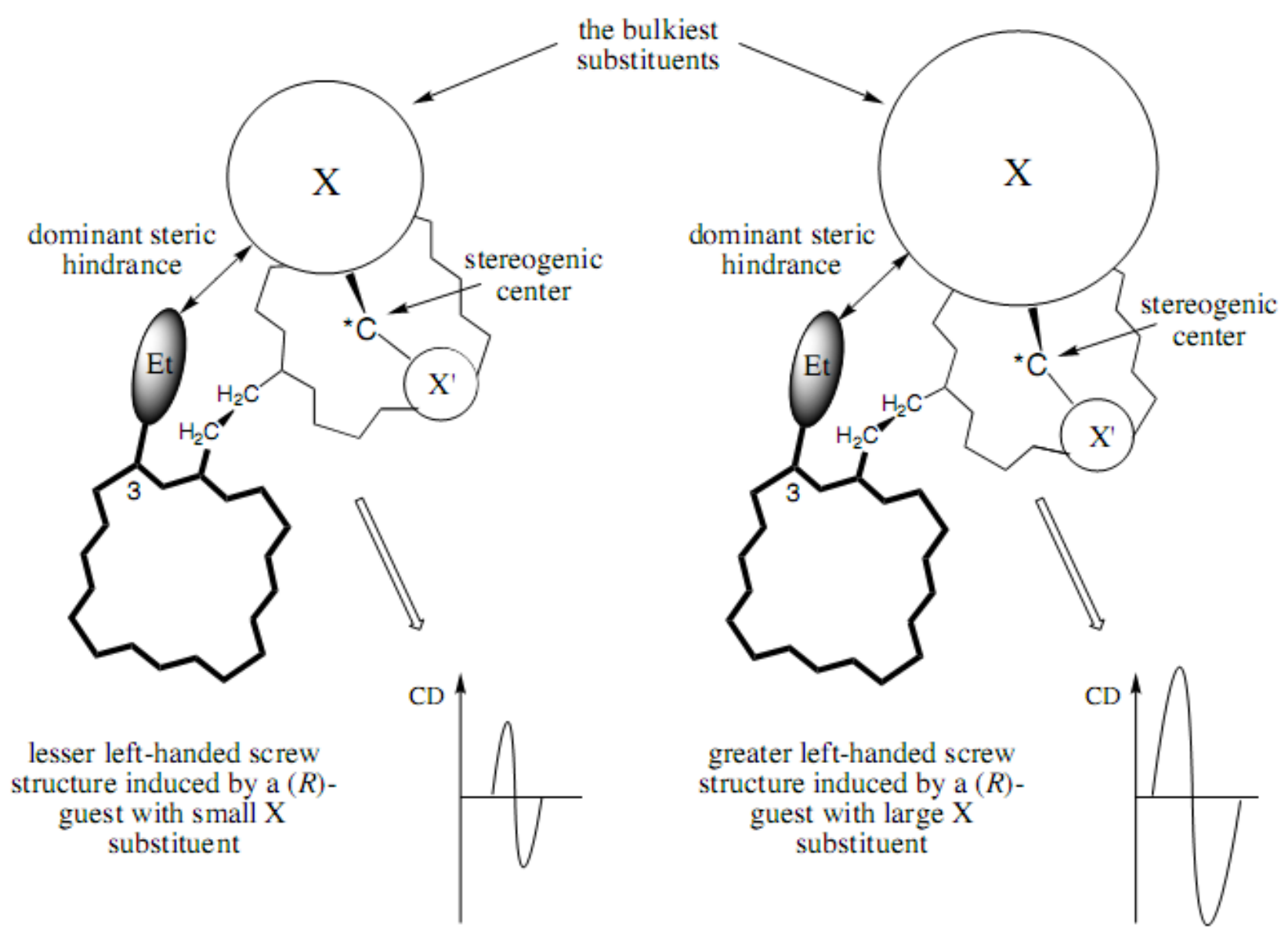

Another essentially important factor controlling chirogenic supramolecular interactions, and thus chirality transfer in these dynamic multicomponent assemblies, was found to be temperature. As could be expected, reduction in temperature significantly enhances the degree of host-guest binding resulting in increasing the number of chiral steric interactions along with a general reduction in thermal molecular motion, and consequently enhancing the chirality induction in $\mathbf{1}$ upon interaction with chiral ligands. For example, as can be clearly seen in Figure 8, variable-temperature CD monitoring of the

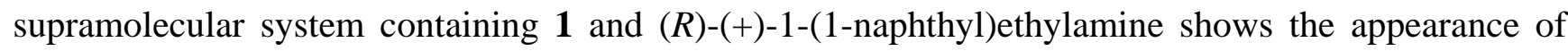
bisignate exciton coupling signal in the absorption region of porphyrin $\mathrm{B}$ band at first that gradually increases as the temperature falls. This temperature-generated enhancement of CD sensitivity allows the chirality induction in the corresponding $\mathrm{Zn}$ complexes (1 or 4) in the presence of considerably smaller amount of a chiral amine (by a factor of 100-1000) or even alcohols that possess a well-known marginal affinity for Zn porphyrins. 
Figure 7. Schematic representation of the solvent effect on chirality induction in the anti conformation of $\mathbf{1}$ upon interaction with monodentate guests.

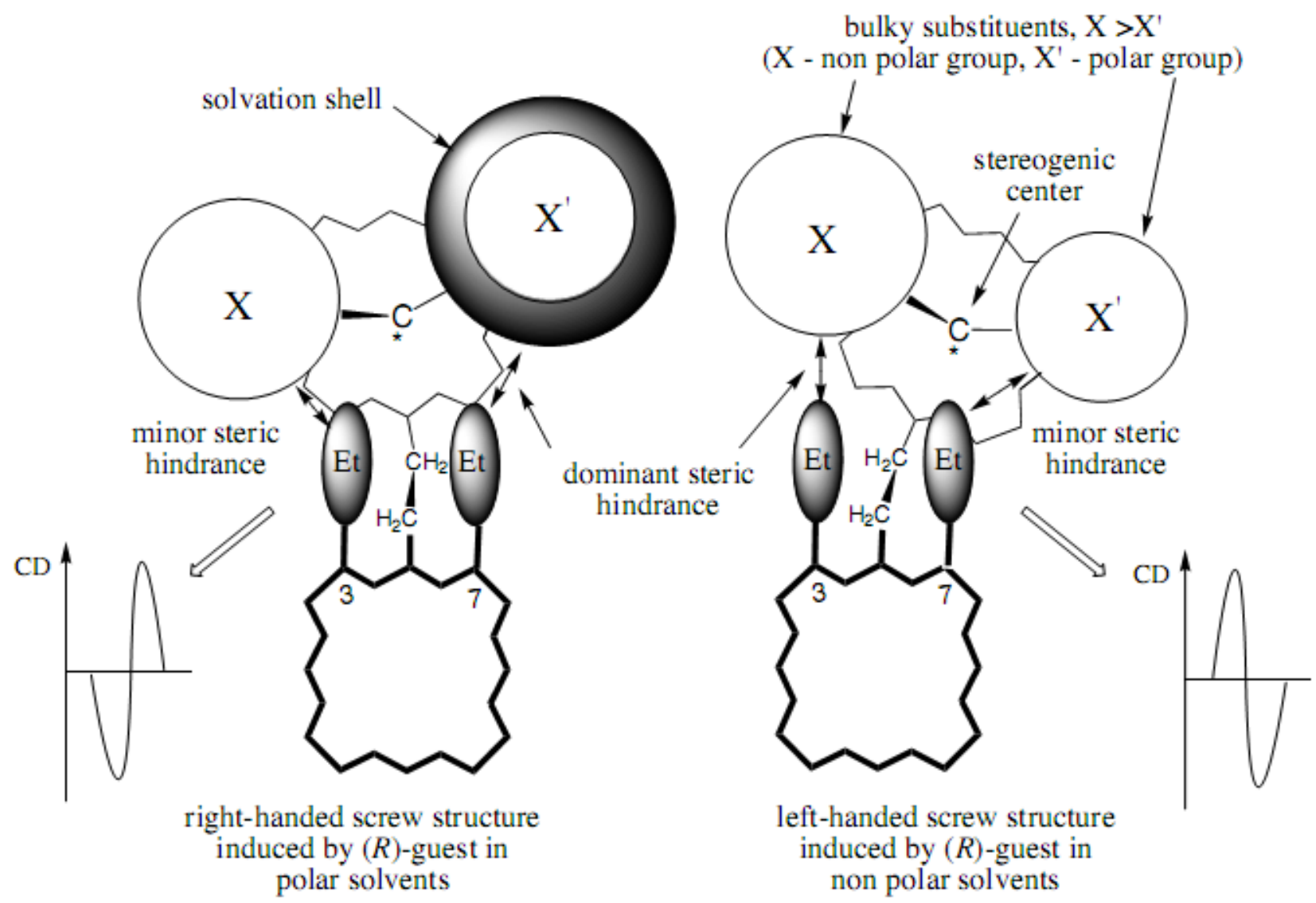

Besides the temperature, the correct selection of the appropriate host-guest pair can be also effectively used as a powerful controlling factor of supramolecular chirogenesis. In particular, one of the attractive features of porphyrins is the relatively facile metal ion insertion into its center. Thus, the appropriate modification of the porphyrin core allows the corresponding selection of host-guest interaction pair to expand the chirogenic universality of the bis-porphyrin structure. In particular, the Mg complex 2 (Figure 2) makes it possible to induce chirality in the bis-porphyrin upon interaction with chiral alcohols even at room temperature, owing to the high affinity of magnesium porphyrins for alcohols. Another good example of the effectiveness of this approach is the metal-free bis-porphyrin 3 (Figure 2), which was efficiently applied for supramolecular chirogenesis generated by various enantiopure acids. Since the chirality induction mechanisms in 1, 2, and $\mathbf{3}$ are essentially similar, despite the difference in host-guest binding, the chiroptical outcome and especially the sign of induced chirality are the same, hence allowing facile and straightforward determination of the absolute configuration of various chiral compounds on the basis of induced CD couplet and affording a versatile chiral sensor on the basis of pattern bis-porphyrin structure. 
Figure 8. Temperature-induced changes in the CD spectrum of 1 in the presence of $(R)$ (+)-1-(1-naphthyl)ethylamine upon cooling from 274 to 152K.

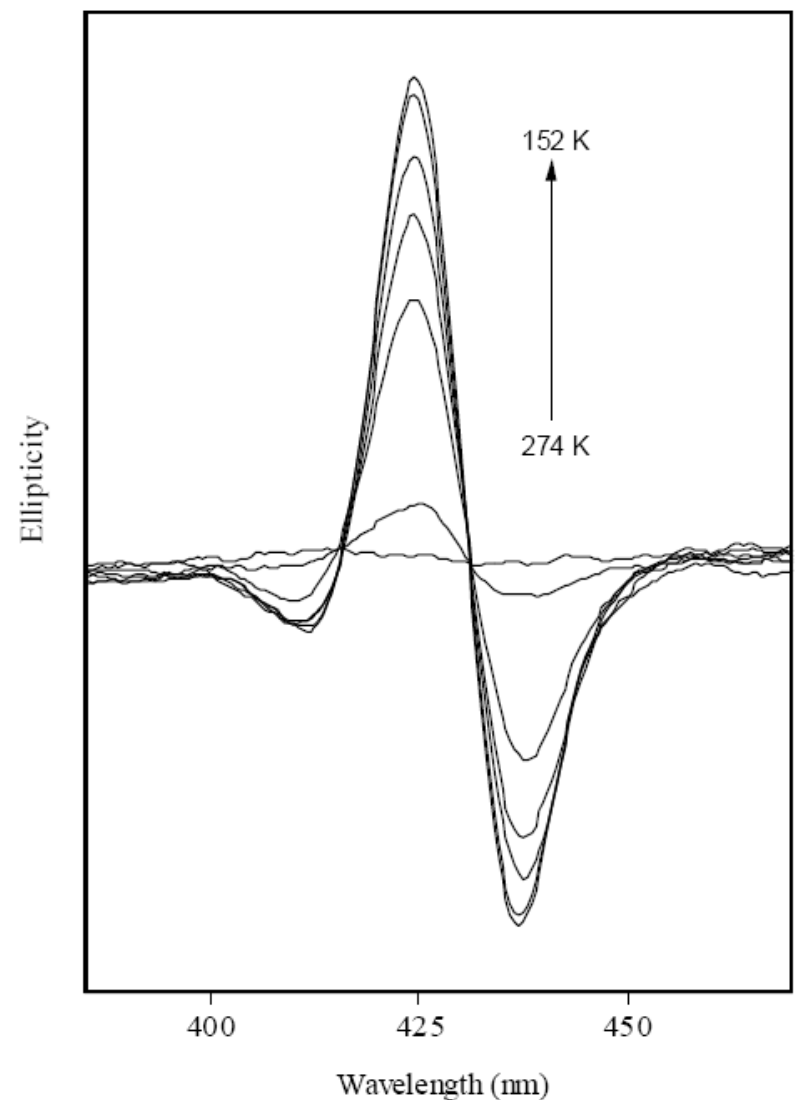

Further chirogenic versatility of this bis-porphyrin structure was nicely demonstrated by interaction of 1 with various chiral bidentate ligands. Besides, this revealed a remarkable stoichiometry effect on the asymmetry transfer mechanism from the chiral guests to the achiral host. Since in $\mathbf{1}$ there are two binding sites, it is energetically favorable for both to be bound. Thus, it was found that on the addition of suitable bidentate guests, extremely stable 1:1 tweezer complexes are formed, in which each of the functional groups of the guest is coordinated to one of the zinc porphyrin centers of the host (Figure 2, see the tweezer case). These tweezer structures are characterized by a significantly enhanced chiroptical activity (with a CD amplitude of more than $500 \mathrm{M}^{-1} \mathrm{~cm}^{-1}$ ) and high association constants (being as high as $>10^{7} \mathrm{M}^{-1}$ ) as compared to those of 1:2 anti species induced by monodentate ligands. This is a result of the higher structural rigidity and more optimal geometry for chirality transfer experienced by these tweezer complexes in comparison to the anti-species due to the concerted nature of the two coordination bonds in the 1:1 complex, rather than the dynamic single coordination bonds of the 1:2 anti complexes. These remarkable properties of the tweezer structures allow a comprehensive and unambiguous rationalization of the induced optical activity in the bis-porphyrinoid based supramolecular system that was achieved for the first time by using enantiopure DACH. In particular, on the basis of the obtained crystallographic data of the 1:1 tweezer structure of 1 with $(R, R)$-DACH and calculations according to the Kuhn-Kirkwood coupled-oscillator mechanism, the induced CD signal in the region of the porphyrin Soret band was assigned to be a combination of the two excitonic homocouplings between the corresponding $\mathrm{B}||$ and $\mathrm{B}_{\perp}$ electronic transitions orientated along the 
porphyrin's meso 5-15 and 10-20 axes, whilst the contribution of the hetero $\mathrm{B} \|-\mathrm{B}_{\perp}$ couplings were found to be negligible. The directions of these two homocouplings were confirmed to be anticlockwise (Figure 9), hence inducing an intense negative CD couplet with an extremely large total amplitude of $-590 \mathrm{M}^{-1} \mathrm{~cm}^{-1}$.

Figure 9. Definition of the directions of the coupling electronic transitions in the 1:1 tweezer complex.

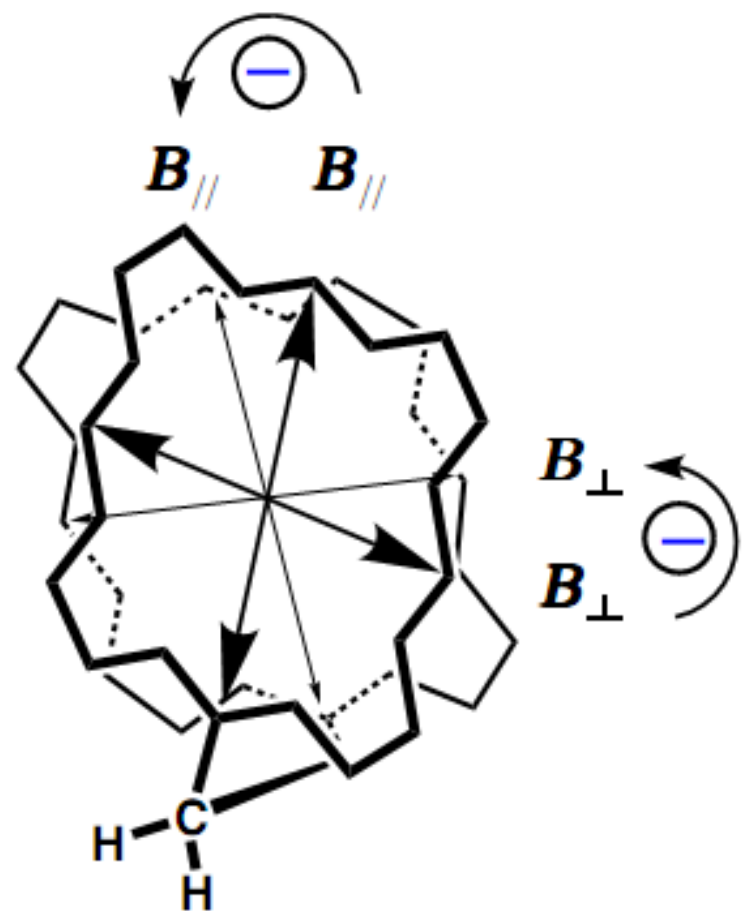

However, in some particular cases, further increasing the concentration of bidentate ligands may shift the supramolecular equilibrium toward the extended 1:2 anti complex. This results in an intriguing phenomenon of chirality inversion based exclusively on the stoichiometry of the supramolecular system, thus allowing comprehensive investigation of the role of the host-guest ratio on the chirality transfer mechanism. For example, upon complexation with enantiomeric 1,2diphenylethylenediamine (DPEA) and L-Thr-OMe, a remarkable chirality switching controlled solely by the stoichiometry of the supramolecular system was observed and assigned to the opposite spatial orientation of the 1:1 and 1:2 complexes (Figure 10). Hence, upon the addition of $(R, R)$-DPEA at the low concentration region, the formation of the tweezer structure is favored with the generation of positive chirality. On further increase of the ligand concentration, transformation to the $1: 2$ anticomplex takes place, accompanied by a chirality inversion process to yield the negative chirality sign. The supramolecular mechanism of this unusual chiroptical effect is a result of the geometry and preorganization of the bidentate guest. Thus, to minimize the host-guest steric repulsive interactions in each conformation, the chirality of the 1:1 tweezer and 1:2 anti-complexes must be opposite. This is a unique example of how it is possible to generate supramolecular chirality into an achiral host and then, while keeping the local chiral information of the inductor constant, invert the overall chirality of the system by simply changing the host/guest stoichiometry. 
Figure 10. Schematic representation of the chirality induction and inversion in $\mathbf{1}$ by bidentate ligands.

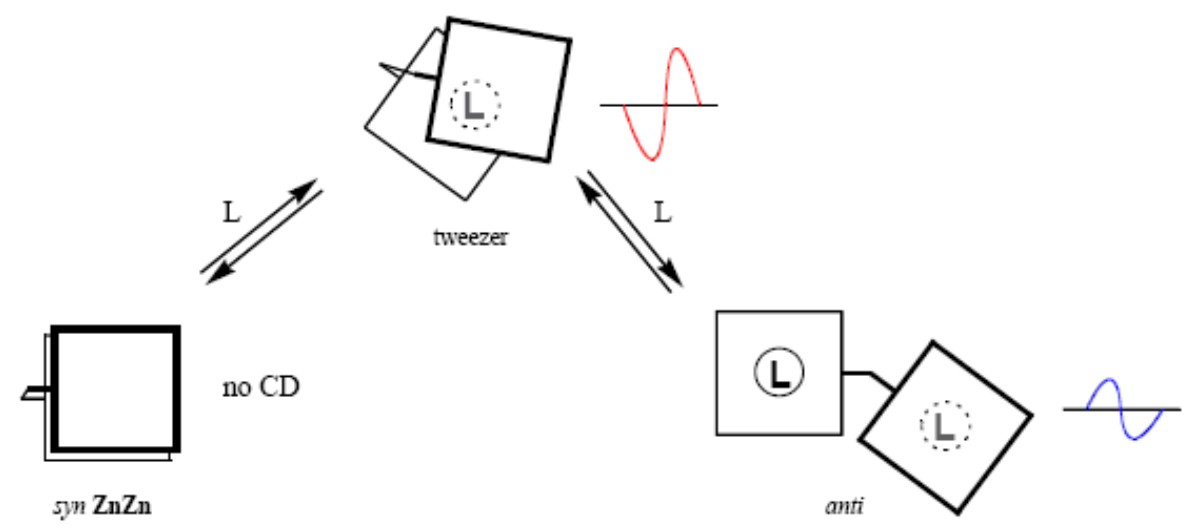

Besides the mentioned above controlling factors, the phase transition itself may also be served as an additional stimulus for supramolecular chirogenesis. As in the case of solution phase, the solid state can possess specific properties and phenomena unique to this particular phase, making it possible to use phase transition as an effective functional tool regulating the chirality induction processes. Therefore, further chirogenic diversity of these bis-porphyrin based systems was expanded to the solid state. In this case, besides the inherent chirogenic steric repulsions within the essentially isolated supramolecular system as observed in solution, the intersystem (that is between two or more individual supramolecular systems) interactions should be also taken into account. In particular, comparison of the corresponding chiroptical properties revealed a remarkable difference between these two phases. For example, in solution, $(R)$-guests induce a negative CD couplet in the B (Soret) band region of $\mathbf{1}$, corresponding to an anticlockwise orientation between the lowest energy $\mathrm{B} / \mid$ electronic transitions. On the contrary, the same $(R)$-guests yield an enhanced positive bisignate CD signal of more complicated profile and shifted to the lower energy in the solid state ( $\mathrm{KBr}$ matrix), indicating anticlockwise orientation of the $\mathrm{B} \|$ transitions. The observed phenomenon of chirality inversion and amplification is a result of numerous intermolecular electronic transition couplings, which are somehow overlapped with the corresponding higher energy intramolecular couplings, in the supramolecular aggregates formed in the solid state (Figure 11). A similar amplification of marginal solution-phase chirality in the solid state was also observed even for a zinc octaethylporphyrin monomeric system in the presence of enantiopure amines [35]. In this case, over a period of 4 days, exceptionally high optical activity with the anisotropy factor being as great as 0.015 was generated, the sign of which was the same as that observed for the solid state systems on the basis of $\mathbf{1}$, indicating essentially the same chirogenic mechanism.

\section{Structural Modifications of the Ethane-Bridged Bis-Porphyrins}

A structural modification of the porphyrin host can be another effective factor to control supramolecular chirogenesis. For example, a simple reduction of one pyrrole ring in each porphyrin subunit of the parent bis-porphyrin yielded a racemic mixture of the corresponding bis-chlorin $\mathbf{5}$ (Figure 12). Further optical resolution resulted in a novel chiral bis-chlorin host, which opens up new prospective possibilities in the field of supramolecular chirogenesis by allowing effective 
enantioselective recognition of various chiral guests [36-38]. Hence, enantiopure 5 exhibits the CD signal reduction upon interaction with chiral guests due to the induced conformational changes, whilst the chiroptical response was found to be noticeably different for the corresponding antipodal amines. The mechanism of chiral recognition is based upon two-point host-guest interactions combined with the variability of the coupling electronic transitions of the chromophoric host, the chiral orientation of which was controlled by the guest's stereochemistry. In particular, as shown above for bis-porphyrins 1-4, the external ligation occurs from both sides of the chlorin ring to give the "inside" and "outside" coordination species, which are in a fast dynamic equilibrium. Upon the outside ligation, the original conformation of 5 remains essentially unchanged due to the lack of direct host-guest steric interactions, thus resulting in minor chiroptical changes. However, the inside binding induces significant conformational changes owing to steric interactions between the guest and the neighboring chlorin moiety of 5 producing the corresponding anti conformation, as in the case of the bis-porphyrin analogues 1-4. The CD response of anti conformation itself is considerably different in comparison to that of original conformation and its helicity is governed by the chirality of external ligand yielding the opposite screw orientation produced by $R$ - and $S$-guests. Since all the resulting conformations are chiral, there is the overall summation of whole existing excitonic interactions resulting in the different degree of the CD amplitude reduction observed for opposite enantiomers, thus allowing recognition of the corresponding antipodes. In conclusion, this innovative approach makes it possible to use $\mathbf{5}$ for the efficient chiral recognition of a wide range of chiral compounds.

Figure 11. Schematic representation of the intra- and inter-molecular exciton couplings in the supra-molecular aggregates of $\mathbf{1}$ formed in the solid state in the presence of $(R)$-ligand.

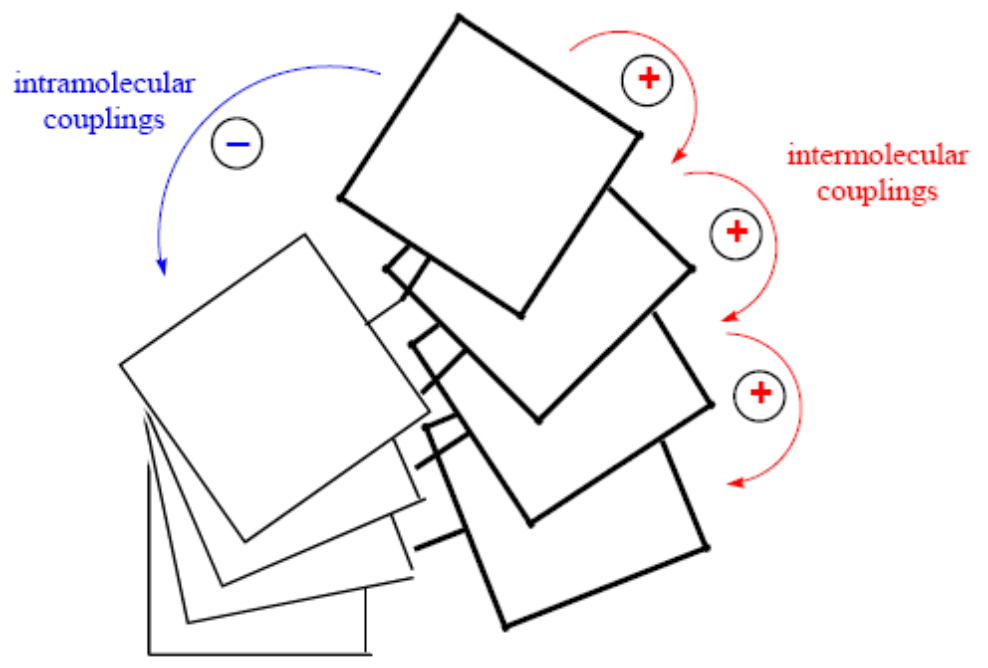

More sophisticated structural modification resulted in a rather complicated achiral tetrameric porphyrin host 6 (Figure 13) [39]. High chirogenic sensitivity of this tetranickel complex allows investigation of the asymmetry induction phenomenon upon extremely weak host-guest interaction modes and marginal chiroptical responses. Thus, for all chiral solvents studied, $(R)$-solvents induced a positive $\mathrm{CD}$ couplet in the region of the long-ranged $\mathrm{B}_{\mathrm{x}}$ electronic transition, whilst (S)-solvents yielded a negative $C D$ couplet, even in the case of $\alpha$-pipene having no functional groups. This achiral host exists in the syn conformation, which is stabilized by the intramolecular porphyrin-porphyrin and 
diacetylene-diacetylene interactions, hence producing a small-sized cavity between two terminal porphyrin moieties. The solvent molecules are able to penetrate inside this cavity to enhance the solvent-solute intermolecular interactions, thus transferring the chiral information from the chiral guests to the achiral host via the unidirectional screw formation in $\mathbf{6}$. The induced helicity is governed by the guest's absolute configuration, whilst the host-guest interactions are apparently based upon van der Waals forces rather than the solvent coordination as evidenced by the well-known property of nickel porphyrins to be inert toward the alcohol ligation and the observed chirality induction by hydroxyl-free solvent. This opens up exciting prospects for chirality sensing of a wide variety of chiral solvents regardless of the corresponding guest's structural consideration.

Figure 12. Structure of bis-chlorin 5.
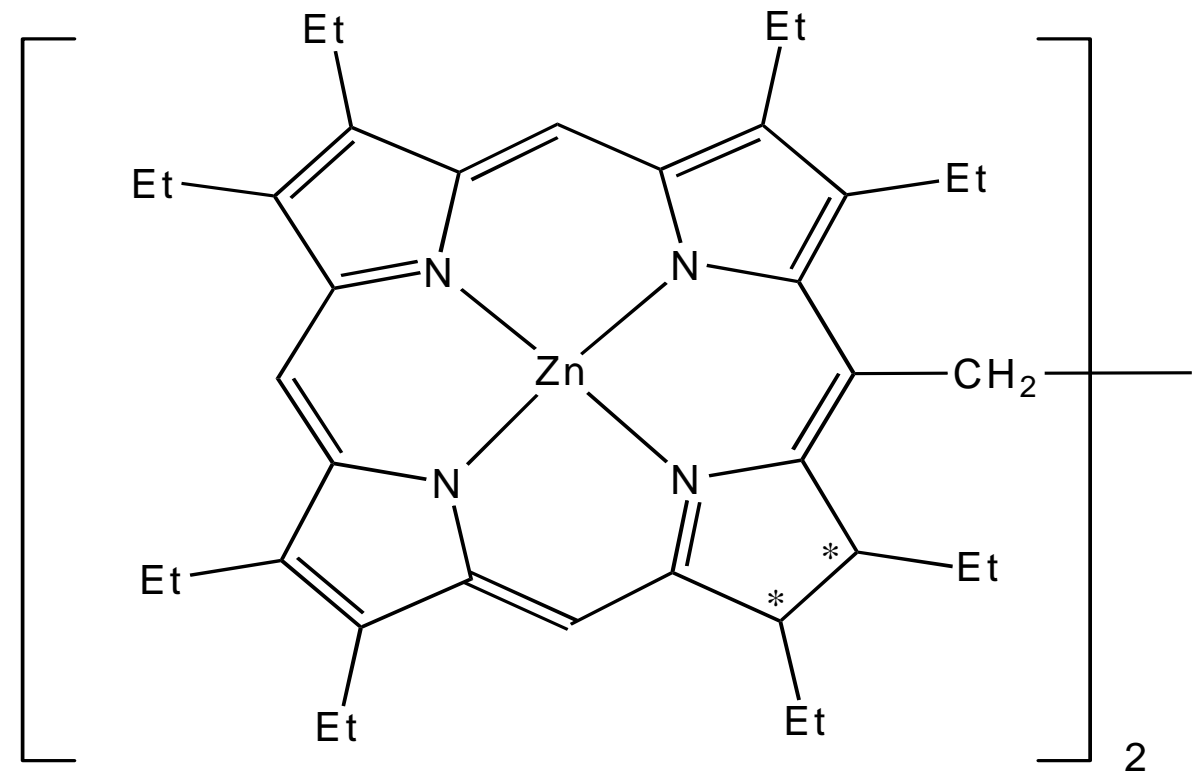

5

Figure 13. Structure of tetrameric porphyrin 6 and orientation of the $B_{x}$ transition.

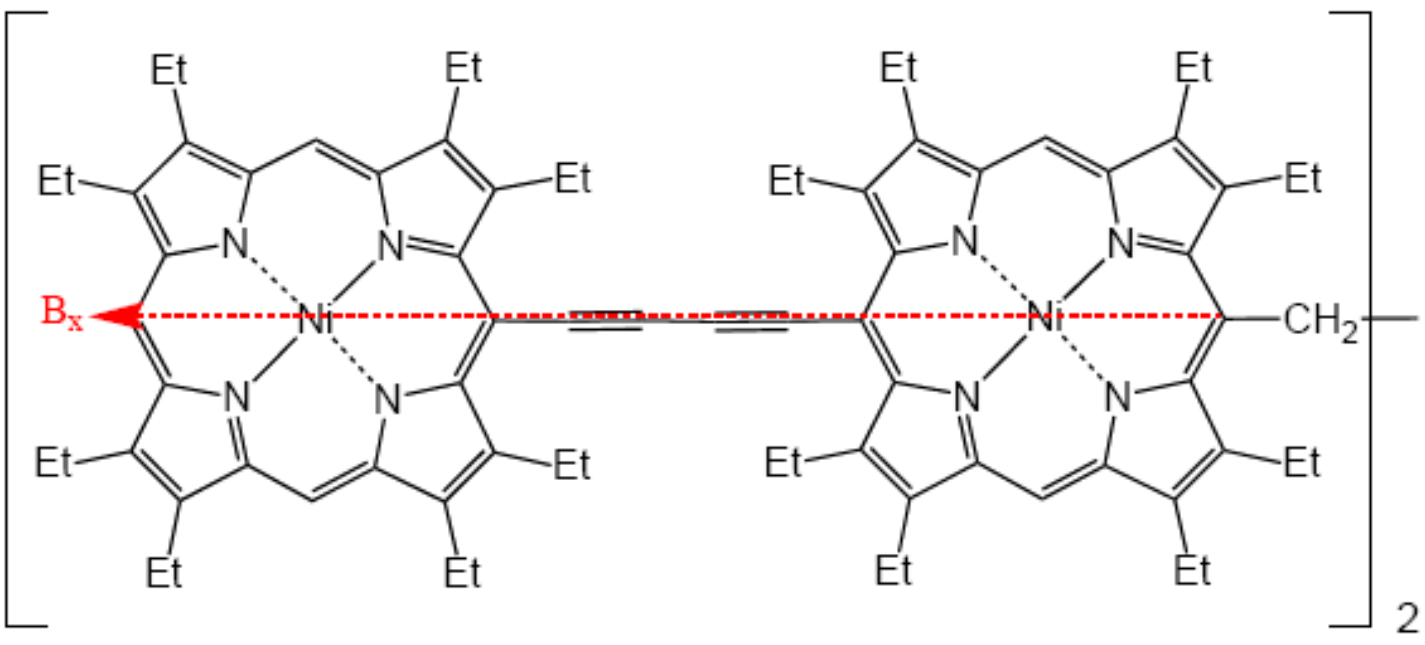




\section{Conclusions}

Since supramolecular chirogenesis is one of the most fundamental and important issues of modern science and advanced technology, understanding of the corresponding driving forces, mechanisms, and various controlling factors is a paramount research goal. Design and development of a universal structural motif of the supramolecular host, which is able to perform various chirogenic tasks, is a smart approach for comprehensive investigation and further application of supramolecular chirogenesis. This feature paper has overviewed recent advances in the design and use of ethanebridged bis-porphyrinoids for diverse applications in the chirality fields and uniquely demonstrated that this structural motif is the most universal chirogenic unit to this end.

\section{References}

1. Voet, D.; Voet, J.G. Biochemistry, 2nd ed.; John Wiley \& Sons, Inc.: New York, NY, USA, 1995.

2. Borovkov, V.V.; Inoue, Y. A versatile bisporphyrinoid motif for supramolecular chirogenesis. Eur. J. Org. Chem. 2009, 189-197.

3. Hembury, G.A.; Borovkov, V.V.; Inoue, Y. Chirality sensing supramolecular systems. Chem. Rev. 2008, 108, 1-73.

4. Mamardashvili, N.Zh.; Borovkov V.V.; Mamardashvili G.M.; Inoue, Y.; Koifman, O.I. Complexation of porphyrins with ions and organic molecules. In Chemical Processes with Participation of Biological and Related Compounds. Biophysical and Chemical Aspects of Porphyrins, Pigments, Drugs, Biodegradable Polymers and Nanofibers; Lomova T.N., Zaikov G.E., Eds.; Koninklijke Brill: Leiden, The Netherlands, 2008; p. 117.

5. Borovkov, V.V.; Inoue, Y. Supramolecular chirogenesis in host-guest systems containing porphyrinoids. Top. Curr. Chem. 2006, 265, 89-146.

6. Borovkov, V.V., Mamardashvili, N.Zh.; Inoue, Y. Optically active supramolecular systems on the basis of porphyrins. Russ. Chem. Rev. 2006, 75, 820-832.

7. Borovkov, V.V.; Hembury, G.A.; Inoue, Y. Origin, control, and application of supramolecular chirogenesis in bis-porphyrin based systems. Acc. Chem. Res. 2004, 37, 449-459.

8. Saenger, W. Principles of Nucleic Acid Structure; Springer: New York, NY, USA, 1984.

9. Steed, J.W.; Atwood, J.L. Supramolecular Chemistry; John Wiley \& Sons, Ltd.: Chichester, UK, 2000.

10. Kyte, J. Structure in Protein Chemistry; Garland Publishing, Inc.: New York, NY, USA, 1995.

11. Borovkov, V.V.; Lintuluoto, J.M.; Inoue, Y. Synthesis of Zn-, Mn- and Fe-containing mono- and heterometallated ethanediyl-bridged porphyrin dimers. Helv. Chim. Acta 1999, 82, 919-934.

12. Borovkov, V.V.; Lintuluoto, J.M.; Inoue, Y. Temperature controlled syn-anti conformational switching in zinc containing porphyrin dimers via ligand assistance. Tetrahedron Lett. 1999, 40, 5051-5054.

13. Borovkov, V.V.; Lintuluoto, J.M.; Inoue, Y. Syn-anti conformational changes in zinc porphyrin dimers induced by temperature-controlled alcohol ligation. J. Phys. Chem. B 1999, 103, 5151-5156. 
14. Borovkov, V.V.; Lintuluoto, J.M.; Fujiki, M.; Inoue, Y. Temperature effect on supramolecular chirality induction in bis (zinc porphyrin). J. Am. Chem. Soc. 2000, 122, 4403-4407.

15. Borovkov, V.V.; Lintuluoto, J.M.; Inoue, Y. Supramolecular chirogenesis in bis(zinc porphyrin): an absolute configuration probe highly sensitive to guest structure. Org. Lett. 2000, 2, 1565-1568.

16. Borovkov, V.V.; Lintuluoto, J.M.; Inoue, Y. Elucidation of the mechanism of supramolecular chirality inversion in bis(zinc porphyrin) by dynamic approach using $\mathrm{CD}$ and ${ }^{1} \mathrm{H}$ NMR spectroscopy. J. Phys. Chem. A 2000, 104, 9213-9219.

17. Borovkov, V.V.; Lintuluoto, J.M.; Inoue, Y. Supramolecular chirogenesis in zinc porphyrins: mechanism, role of guest structure, and application for the absolute configuration determination. J. Am. Chem. Soc. 2001, 123, 2979-2989.

18. Borovkov, V.V.; Yamamoto, N.; Lintuluoto, J.M.; Tanaka, T.; Inoue, Y. Supramolecular chirality induction in bis(zinc porphyrin) by amino acid derivatives: rationalization and applications of the ligand bulkiness effect. Chirality 2001, 13, 329-335.

19. Borovkov, V.V.; Lintuluoto, J.M.; Inoue, Y. Stoichiometry-controlled supramolecular chirality induction and inversion in bis-porphyrin systems. Org. Lett. 2002, 4, 169-171.

20. Borovkov, V.V.; Lintuluoto, J.M.; Sugeta, H.; Fujiki, M.; Arakawa, R.; Inoue, Y. Supramolecular chirogenesis in zinc porphyrins: equilibria, binding properties, and thermodynamics. J. Am. Chem. Soc. 2002, 124, 2993-3006.

21. Borovkov, V.V.; Harada, T.; Inoue, Y.; Kuroda, R. Phase-sensitive supramolecular chirogenesis in bis-porphyrin systems. Angew. Chem. Int. Ed. 2002, 41, 1378-1381.

22. Borovkov, V.V.; Lintuluoto, J.M.; Sugiura, M.; Inoue, Y.; Kuroda, R. Remarkable stability and enhanced optical activity of a chiral supramolecular bis-porphyrin tweezer in both solution and solid state. J. Am. Chem. Soc. 2002, 124, 11282-11283.

23. Lintuluoto, J.M.; Borovkov, V.V.; Inoue, Y. Direct determination of absolute configuration of monoalcohols by bis(magnesium porphyrin). J. Am. Chem. Soc. 2002, 124, 13676-13677.

24. Borovkov, V.V.; Hembury, G.A.; Inoue, Y. Enthalpy-entropy compensation upon syn-anti conformational switching of bis-porphyrins by amines and alcohols. J. Porphyrins Phthalocyanines 2003, 7, 337-341.

25. Borovkov, V.V.; Lintuluoto, J.M.; Hembury, G.A.; Sugiura, M.; Arakawa, R.; Inoue, Y. Supramolecular chirogenesis in zinc porphyrins: interaction with bidentate ligands, formation of tweezer structures, and the origin of enhanced optical activity. J. Org. Chem. 2003, 68, 7176-7192.

26. Borovkov, V.V.; Hembury, G.A.; Yamamoto, N.; Inoue, Y. Supramolecular chirogenesis in zinc porphyrins: Investigation of zinc-freebase bis-porphyrin, new mechanistic insights, extension of sensing abilities and solvent effect. J. Phys. Chem. A 2003, 107, 8677-8686.

27. Borovkov, V.V.; Hembury, G.A.; Inoue, Y. The origin of solvent-controlled supramolecular chirality switching in a bis(zinc porphyrin) system. Angew. Chem. Int. Ed. 2003, 42, 5310-5314.

28. Borovkov, V.V.; Fujii, I.; Muranaka, A.; Hembury, G.A.; Tanaka, T.; Ceulemans, A.; Kobayashi, N.; Inoue, Y. Rationalization of supramolecular chirality in a bis-porphyrin system. Angew. Chem. Int. Ed. 2004, 43, 5481-5485. 
29. Borovkov, V.V.; Casilli, S.; Conoci, S.; Inoue, Y.; Sortino, S.; Valli, L. Molecular organization and syn-anti conformational equilibria in ethane-bridged bis(zinc porphyrin) floating films at the air-water interface. Surf. Sci. 2004, 572, 66-76.

30. Valli, L.; Casilli, S.; Giotta, L.; Pignataro B.; Conoci, S.; Borovkov, V.V.; Inoue, Y.; Sortino, S. Ethane-bridged zinc-porphyrin dimers in Langmuir-Shäfer thin films: structural and spectroscopic properties. J. Phys. Chem. B 2006, 110, 4691-4698.

31. Fujii, I.; Borovkov, V.V.; Inoue, Y. Crystal structure of bis-Zn-porphyrin. Anal. Sci. 2006, 22, x77-x78.

32. Bhyrappa, P.; Borovkov, V.V.; Inoue, Y. Supramolecular chirogenesis in bis-porphyrins: Interaction with chiral acids and application for the absolute configuration assignment. Org. Lett. 2007, 9, 433-435.

33. Smith, M.B.; March, J. March's Advanced Organic Chemistry: Reactions, Mechanisms, and Structure, 5th ed.; John Wiley \& Sons, Inc.: New York, NY, USA, 2001.

34. Harada, N.; Nakanishi, K. Circular Dichroism Spectroscopy Exciton Coupling in Organic Stereochemistry; University Science Books: Mill Valley, CA, USA, 1983.

35. Borovkov, V.V.; Harada, T.; Hembury, G.A. Inoue, Y.; Kuroda, R. Solid state supramolecular chirogenesis: High optical activity and gradual development of zinc octaethylporphyrin aggregates. Angew. Chem. Int. Ed. 2003, 42, 1746-1749.

36. Borovkov, V.V.; Muranaka, A.; Hembury, G.A.; Origane, Y.; Ponomarev G.V.; Kobayashi, N.; Inoue, Y. Chiral bis-chlorin: Effective enantiomer resolution and absolute configuration determination. Org. Lett. 2005, 7, 1015-1018.

37. Borovkov, V.V.; Hembury, G.A.; Inoue, Y. Supramolecular chirogenesis with bis-chlorin versus bis-porphyrin hosts: peculiarities of chirality induction and modulation of optical activity. J. Org. Chem. 2005, 70, 8743-8754.

38. Borovkov, V.V.; Inoue, Y. Supramolecular chiral recognition by bischlorins: A two-point interaction mode combined with the host's conformational modulation controlled by the guest's stereochemistry and bulkiness. Org. Lett. 2006, 8, 2337-2340.

39. Borovkov, V.V.; Yamamoto, T.; Higuchi, H.; Inoue, Y. Supramolecular chirogenesis in weakly interacting hosts: Role of the temperature, structural and electronic factors in enhancement of chiroptical sensitivity. Org. Lett. 2008, 10, 1283-1286.

(C) 2010 by the authors; licensee Molecular Diversity Preservation International, Basel, Switzerland. This article is an open-access article distributed under the terms and conditions of the Creative Commons Attribution license (http://creativecommons.org/licenses/by/3.0/). 